\title{
Collocare le abilità produttive all'interno di una prospettiva autenticamente comunicativa
}

\author{
PAOLO TORRESAN \\ Centro di Ricerca sulla Didattica delle Lingue \\ Università Ca' Foscari, Venezia
}

\section{Riassunto}

Parlare e scrivere sono, si direbbe, due attività all'ordine del giorno in un'epoca che si dichiara comunicativa. Orbene, è veramente così? In effetti, se è merito dell'approccio comunicativo aver riabilitato le abilità ricettive, esso non ha parimenti assegnato alle abilità produttive, specie la scrittura, il posto che meritano all'interno del processo di apprendimento di una lingua seconda.

Nell'articolo delineiamo una metodologia dell'espressione orale e scritta all'interno di una prospettiva comunicativa capace di equilibrio tra abilità e competenze -una prospettiva, diremmo, autenticamente comunicativa. Rispondiamo alle domande: perché è importante parlare e scrivere liberamente? Quale ruolo può avere l'espressione orale/scritta in riferimento ai processi di comprensione e all'affinamento della competenza metalinguistica?

Nella seconda parte passiamo in rassegna una serie di attività volte a facilitare/potenziare la scrittura e l'oralità.

Parole-chiave: approccio comunicativo, espressione scritta, espressione orale, scaffolding

\section{Resumen}

Se diría que hablar y escribir son dos actividades de primer orden en la actualidad, en una época declarada comunicativa. ¿Será realidad esta afirmación? En efecto, es un merito del enfoque comunicativo, por haber revitalizado las destrezas receptivas, lo que no es igualmente asignado a las destrezas productivas, como es la escritura, lugar que sobresale dentro del proceso de aprendizaje de una segunda lengua. En el artículo se señala una metodología para la expresión oral y escrita dentro de una perspectiva comunicativa capaz de lograr un equilibrio entre destrezas y 
competencias; una perspectiva, diríamos, comunicativa auténtica. Respondamos a algunas preguntas: ¿Por qué es importante hablar y escribir libremente? ¿Qué papel puede tener la expresión oral/escrita con referencia a los procesos de comprensión y al logro de la competencia metalingüística? En la segunda parte pasamos a destacar una serie de actividades dirigidas a facilitar o potenciar la escritura y la oralidad.

Palabras claves: enfoque comunicativo, expresión escrita, expresión oral, andamiaje

\section{I caratteri di una prospettiva autenticamente comunicativa}

$\mathrm{I}$

n questa sezione, dopo una rapida critica a uno tra i limiti maggiori del comunicativismo krasheniano, -l'aver messo in ombra la grammatica, fino a considerarla una dimensione superflua in ordine all'acquisizione di una lingua seconda- sosteniamo che esso opera una impropria distinzione di valore all'interno delle abilità comunicative, a vantaggio di quelle ricettive.

Ne esce un quadro di riferimento più equilibrato, a cui diamo il titolo di prospettiva autenticamente comunicativa.

\subsection{La questione della grammatica}

Gli insegnanti che si ispirano al metodo comunicativo danno valore all'uso della lingua rispetto ad uno studio formale: ritengono più importanti la scorrevolezza e l'efficacia comunicativa (il fatto di farsi capire) rispetto all'accuratezza.

Lo slogan che si diffonde in Europa, successivamente al libro The Communicative Approach to Language Teaching (1978), curato da Christopher Brumfit e Keith Johnson, potrebbe sintetizzarsi nella frase "effective but defective communication": è più importante che lo studente si faccia capire e raggiunga gli scopi comunicativi che si è prefissato rispetto al fatto che si esprima correttamente (cfr. Humphris in Guastalla 2008).

Come ben sintetizza Humphris (2005), lo spostamento di paradigma, da un metodo centrato sulle forme, come lo strutturalismo, ad uno che punta maggiormente ai significati, qual è appunto quello comunicativo, è notevole e può essere descritto secondo vari livelli (lo schema qui sotto è tratto da Humphris 2005, con un leggero riadattamento):

\begin{tabular}{ll}
\multicolumn{1}{c}{ FOCUS SULLE FORME } & FOCUS SUI SIGNIFICATI \\
\hline accuratezza & fluenza \\
consapevolezza linguistica & pratica comunicativa
\end{tabular}


studio della lingua

esercizio delle abilità

attività precomunicative

attività comunicative

La rivalutazione dei significati operata in seno al metodo comunicativo diventa molto rigida in Krashen.

Lo studioso statunitense, che assume il ruolo di portabandiera dell'ala estrema del metodo comunicativo (il comunicativismo), sostiene che un'educazione linguistica basata sulle forme (nei suoi termini: apprendimento) è una forma labile di ritenzione delle informazioni: lo studente che apprende può superare un test grammaticamente orientato ma non possiede una competenza da esibire in contesti non controllati (per esempio, in una conversazione con un madrelingua). All'apprendimento Krashen oppone un'educazione basata sulla compensione dei significati, chiamandola acquisizione. L'acquisizione è un processo inconscio e duraturo. Il soggetto competente è colui che ha acquisito la lingua, non colui che l'ha appresa (Krashen 1981). Tra lo strutturalismo e il comunicativismo si apre una voragine, una distanza incolmabile. Da un lato, nelle classi di lingua degli anni sessanta (ma anche oggi, visto che esiste uno strutturalismo perenne), la lingua è concepita come una serie di strutture, graduate per complessità, a cui lo studente va esposto (gli si mostra la regola) e che è tenuto, in un secondo momento, a reimpiegare mediante esercizi di completamento ("completa la frase con il pronome esatto") o di trasformazione ("volgi la frase al passato"). Dall'altro, Krashen e quanti si riconoscono nel suo paradigma, danno un'importanza minima allo studio e all'esercizio delle forme. Krashen piuttosto ritiene che le forme vengano acquisite solo se inglobate in un testo che risulti comprensibile allo studente. Esercitarsi sulla grammatica a prescindere dall'input non ha alcun impatto -secondo Krashen- sul consolidarsi della competenza (cfr. Krashen 1983).

In realtà, benché opposti su molte istanze, lo strutturalismo e il comunicativismo operano una stessa azione: separano due momenti dell'apprendimento linguistico: la comunicazione, da un lato, e l'analisi/reimpiego delle forme, dall'altro, dichiarando la bontà dell'uno, in un caso, e dell'altro, nel secondo.

È possibile però pensare a una posizione che concili gli estremi, che chiamiamo autenticamente comunicativa (d'ora in poi PAC), nel tentativo di riunire, in un unico quadro di riferimento metodologico, competenze e abilità, sapere la lingua (know what) e saper fare con la lingua (know how).

In una PAC né la grammatica né la comunicazione hanno una posizione ancillare, dal momento che esiste la consapevolezza che la grammatica senza la comunicazione produce un soggetto incapace di interagire con gli altri, mentre la comunicazione senza la grammatica conduce a scarsa consapevolezza metalinguistica $^{1}$ e a fossilizzazioni ${ }^{2}$.

Più in particolare, è difficile che gli studenti allenati ad un metodo strutturalista possano (Nunan 2001: 67;la traduzione è nostra)

fare delle connessioni tra diverse parti dei sistemi grammaticali. È altrettanto difficile [da parte loro] valutare come applicare la grammatica 
imparata al fine di comunicare. [Nello strutturalismo] si imparano le parole come pezzi staccati, mediante liste; gli allievi non possono, di conseguenza, sviluppare una comprensione di come esse siano raggruppate, a seconda del loro significato, in campi semantici”.

Nello strutturalismo, continua Nunan, si nutre la convinzione che (la traduzione è nostra)

acquisire una seconda lingua sia un processo lineare, tale per cui gli allievi imparano un pezzo alla volta, affrontando l'unità elementare dapprima, per poi, passo dopo passo, affrontare unità più complesse.

Lo strutturalismo è dunque una visione semplificata della lingua e dell'apprendimento; comunicare in lingua, in realtà, implica la gestione simultanea di diverse sottoprocedure (morfosintattiche, semantiche, fonetiche, ortografiche, pragmatiche); gli studenti, "allo stesso tempo, imparano diverse cose in maniera imperfetta” (Nunan 2001: 69).

Imparare una lingua è, inoltre, un processo dinamico, di strutturazione e ristrutturazione della conoscenza; c'è una certa ingenuità -nell'ottica di Nunanin coloro che suppongono sia invece un fenomeno lineare, sommativo, per accumulo di dati.

Il soggetto impara una lingua mediante processi di osservazione (noticing; Schmidt 1990) e di formulazione di ipotesi -ipotesi che, a loro volta, sono soggette a una validazione e una proceduralizzazione i (cfr. McLaughlin 1987).

Il soggetto osserva, fa ipotesi e cerca la conferma delle stesse: questa è la validazione. Confermate le ipotesi, per via di un confronto con l'input autentico, e sedimentate mediante la ripetizione, nella memoria di chi impara si libera, per così dire, dello spazio affinché abbiano luogo nuovi processi induttivi, secondo il ciclo appena descritto: questa è la proceduralizzazione.

La tradizione glottodidattica veneziana, ispirandosi alla Gestalt (cfr. Freddi 1979; Balboni 1994; 2007; Caon 2005), insiste nel ribadire che tale azione dinamica avviene secondo una sequenza ben precisa: GAS, globalità-analisi-sintesi. Lo studente, cioè, esposto ad un testo, si chiede conto del significato (cosa?); successivamente, riflette sulle forme (come?); in terzo luogo, rielabora le stesse in un contesto significativo, al fine di vederle confermate nell'interazione con l'interlocutore.

Il momento dell'analisi, secondo questa sequenza, è una tappa obbligata, che ogni studente compie, anche in un contesto spontaneo. Nella mente di ogni persona esposta ad un input in LS, si origina una varietà linguistica soggetta a mutazione, in un processo di avanzamento e regressione, caratterizzato da curve a U, come nell'immagine qui sotto rappresentata (Pallotti 2005: 50), relativa alla stabilizzazione del participio irregolare del verbo prendere (fig. 1). 
Percorsi a U (tratta da Pallotti 2005: 50)

\section{Percorsi a U}

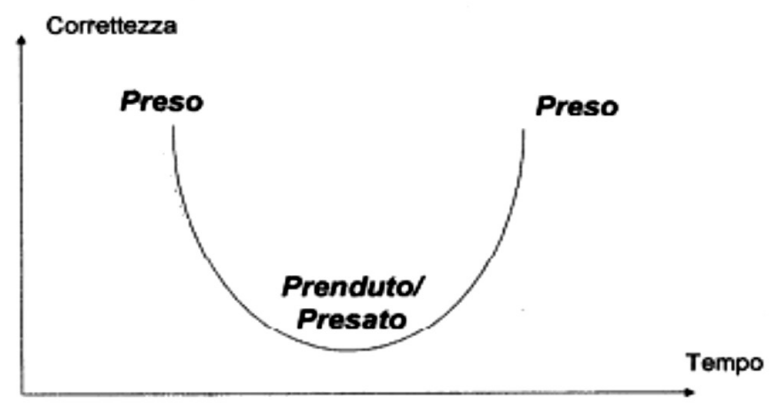

Pallotti commenta così il grafico (2005: 50, il corsivo è nostro):

Nella fase iniziale "preso» era probabilmente una forma fissa, imparata a memoria, mentre "presato» dimostra invece la consapevolezza di una regola che viene sovraestesa. È probabile che l'apprendente abbia acquisito "preso» come forma basica del verbo "*presare», che al passato fa appunto «preso». Ora, basandosi sulla rappresentazione dell'apprendente del lessema "prendere», cioè «*presare», si hanno delle informazioni importanti relative alla sua grammatica, che contempla una regola per formare il passato, una regola produttiva, che rappresenta un passo in avanti rispetto alla forma iniziale, per quanto corretta. Successivamente egli imparerà che "prendere» è un verbo con un paradigma irregolare che alcune volte esce in "prend-» e altre volte esce in "pres-» a seconda del tempo e poi che "preso» è un'eccezione. Concludendo, il percorso seguito dall'apprendente è il seguente: si passa da una prima fase di apprendimento mnemonico di una forma, a una seconda fase di verifica delle ipotesi su quella forma, a una terza fase in cui vi è la regolarizzazione della forma.

Percorsi guidati di analisi delle forme non fanno altro che velocizzare e ottimizzare un fenomeno a cui lo studente contribuisce già di per sé: la costruzione della sua interlingua, ovvero della sua varietà personale di lingua, a metà strada tra la lingua madre e la lingua obiettivo. La riflessione sulle forme, insomma, sostiene e potenzia i processi spontanei di formazione della competenza metalinguistica (cfr. Balboni 1997); in assenza di tale riflessione, la lingua appresa in contesto spontaneo da apprendenti adulti può andare incontro a fossilizzazioni ${ }^{3}$.

Una PAC, quindi, riabilita la grammatica (centrale ed esclusiva nello strutturalismo), presentandola, tuttavia, come un momento subordinato alla comprensione dei testi. 


\subsection{La riabilitazione delle abilità produttive}

La PAC implica, peraltro, una 'democratizzazione' delle abilità comunicative.

Per Krashen leggere e ascoltare sono condizioni necessarie e sufficienti per acquisire lingua. Di conseguenza, nell'ottica comunicativista, le abilità ricettive (leggere e ascoltare) sono più importanti di quelle produttive (parlare e scrivere).

Altri autori sono invece andati manifestando la imprescindibilità dell'output ai fini della costruzione della competenza linguistico-comunicativa, dal momento che esprimersi in un altro codic:

a. consente allo studente di verificare la tenuta dellinterlingua), gli permette cioè di monitorare l'assimilazione delle regole (Swain 1985; Izumi 1999; 2002);

b. se associato a testi oggetto di comprensione, diventa, in sede di interazione tra pari, una potente strategia di approfondimento della comprensione stessa (Long 1991; 1996);

c. fa si che lo studente si riconosca parte attiva del processo di acquisizione, capace di azione sulla lingua e mediante la lingua (Vigil, Oller 1976).

Soffermiamoci sui ciascuno dei tre punti.

\section{Punto a}

Sulla scia di una riflessione di Krashen (1983: 66), Swain afferma (1991: 239; la traduzione è di M. C. Coonan, in id. 2002: 188):

In molti casi noi utilizziamo la sintassi per comprendere -spesso cogliamo il messaggio attraverso una combinazione di lessico, oppure lessico e informazioni extralinguistiche.

In altri termini la comprensione può appoggiarsi sul lessico, in parte sulla sintassi e altre variabili extralinguistiche, bypassando la morfologia.

Nell'output, è bene notare, questo 'evitamento' non è possibile (1991: 240; traduzione come sopra):

Possiamo comprendere un discorso senza conoscere precise regole sintattiche e morfologiche, ma non possiamo produrre discorso con accuratezza senza la conoscenza precisa sintattica e morfologica.

Le abilità produttive implicano, difatti, il consolidarsi delle regole (analizzate); contribuiscono cioè all'evolversi della grammatica interna (formatasi in virtù di un processo di osservazione spontaneo).

Circa il rapporto tra competenza metalinguistica e uso della lingua, Dabrowska, neurobiologa del linguaggio, scrive (2004: 27; il corsivo e la traduzione sono nostri): 
La nostra sensibilità alla frequenza [ovverosia a quanto un certo dato linguistico si ripete all'interno di un testo, ndt.] ci rivela come la rappresentazione mentale del linguaggio [quindi la competenza metalinguistica, ovvero la capacità di astrarre le regole, ndt.] sia modellata dall'uso della lingua stessa [ovvero dal fatto di produrre lingua, ndt.] ed è quindi controproducente delineare una netta distinzione tra la competenza [la conoscenza dichiarativa della lingua, il fatto di conoscerne la grammatica, ndt.] e la performance [la capacità di fare lingua, di usare una lingua per realizzare determinati scopi, quindi la conoscenza procedurale della lingua, ndt.]

Grammatica e uso della lingua sono legati l'uno all'altro. Conoscere la grammatica di una lingua permette di possederla, di esercitare un controllo; specularmente, la stessa competenza metalinguistica viene migliorata dal fatto di usare la lingua ${ }^{4}$.

Scopriamo così che i momenti tenuti separati da strutturalismo e comunicativismo-grammatica e comunicazione-sono, in realtà, vincolati reciprocamente.

\section{Punto b}

Le abilità comunicative consentono di migliorare la comprensione di un testo. In altri termini, la comprensibilità dell'input dipende dalle occasioni di ritorno sul testo (Humphris 1982b) e, soprattutto, dalle opportunità di rielaborazione dei contenuti accordate alla classe (Long 1991; 1996).

Humphris ha ideato, a riguardo, un'attività in cui input e output sono strettamente collegati e l'ha posta al centro del suo manuale di italiano per stranieri, Volare: l'ascolto/lettura ripetuti (Catizone et al. 1997).

L'attività consiste in letture/ascolti reiterati di un brano relativamente complesso. Nel caso della comprensione scritta, la lettura viene eseguita ad una velocità pari a quella di un madrelingua. Tra un ascolto/una lettura e l'altro/a, lo studente scambia le informazioni (idee, ipotesi, dubbi, ecc.) con un compagno di volta in volta diverso.

Il processo che si intende promuovere è quello di un gap filling progressivo, mediato da scambi e finalizzato ad una perlustrazione profonda dei significati. Si vuole raggiungere una equalizzazione delle zone di sviluppo prossimale, secondo una prospettiva tridimensionale: anziché appartenere al testo, la comprensibilità è frutto della relazione tra studente e testo; è quindi suscettibile di avanzamenti, mano a mano che lo studente ritorna sul testo e condivide le ipotesi con altri (cfr. Humphris 1982a; 1982b; 1982c; 1988).

\section{Conseguenze dei punti a) e b) a livello di teoria dell'apprendimento linguistico}

Al fine di rappresentare il processo di apprendimento linguistico, Pallotti (2002: 18) riprende da Ellis (1998) il seguente schema, apportandovi leggeri adattamenti (fig. 2): 
Figura 2

La sequenza dei processi di acquisizione (tratta da Pallotti 2002: 18)

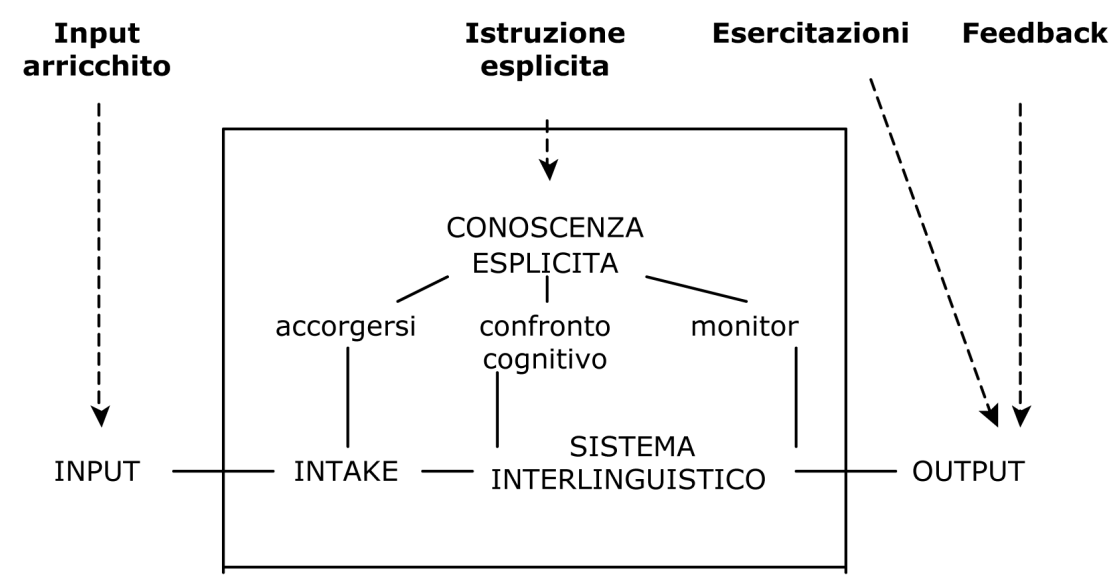

Pallotti spiega l'immagine con queste parole (2002: 18):

Nella parte inferiore della figura viene mostrato il percorso di acquisizione, quale può verificarsi anche in assenza di interventi didattici (il riquadro racchiude processi che avvengono nella mente dell'apprendente). L'input ricevuto è solo in parte trattenuto nella memoria di servizio, diventando così intake, quella parte dell'input su cui l'apprendente compie qualche tipo di operazione cognitiva; altri aspetti dell'input non vengono invece nemmeno notati. L'intake è il materiale con cui viene formato e ristrutturato il sistema interlinguistico, grazie al quale è possibile produrre nuove frasi nella seconda lingua (l'output). La conoscenza esplicita della L2 può intervenire a vari livelli. In primo luogo può favorire l' "accorgersi" di certe strutture nell'input, facendo sì che queste entrino a far parte dell'intake. Poi può far "notare una differenza" tra queste strutture e quelle prodotte dal sistema interlinguistico: attraverso questo confronto cognitivo si avvia la ristrutturazione dell'interlingua, in direzione della lingua d'arrivo. La conoscenze esplicita serve infine per "monitorare" le proprie frasi prima di produrle apertamente: uno potrebbe ad esempio controllare se una frase ancora a livello di "linguaggio interiore" risponda a certe regole apprese esplicitamente.

Pallotti riassume, così, i termini validazione e proceduralizzazione di cui abbiamo parlato in precedenza.

Ora, lo schema di Ellis ricolloca la grammatica (e quindi l'istruzione formale) all'interno del processo di acquisizione linguistica, in aperta polemica alla defenestrazione operata da Krashen. È il primo passo della PAC, abbiamo detto. 
Tuttavia, il processo di apprendimento è rappresentato come lineare, cosa che invece non è. Lo stesso Pallotti, abbiamo visto poc'anzi, sottolinea il fatto che l'acquisizione implica retrocessioni e aggiustamenti (cfr. § 1.1).

Stando alle tesi degli autori dei punti a) e b) -i quali appunto ritengono che, nell'atto di produrre, lo studente consolidi la competenza metalinguistica e la comprensione- possiamo anzi torcere la linea all'altezza dell'output, e costringerla a ritornare indietro, per recuperare forme e significati.

L'output, abbiamo precisato infatti, ha conseguenze tanto sull'input quanto sul sistema interlinguistico: rende più trasparente l'uno e più solido l'altro. Pertanto, esso non è l'estremo della retta ma è il punto in cui la retta si fa curva e si delinea una circonferenza. Ricorrendo a una metafora sportiva, potremmo dire che l'output non è la tappa finale di un percorso ciclistico del tipo: ParigiRoubaix, ma di un circuito del tipo: Parigi-Roubaix-Parigi. Nell'atto di varcare il traguardo (Roubaix), si ripercorrono le tappe precedenti. È come un film il cui finale permette di chiarire il contesto e le intenzioni dei personaggi, costringendo lo spettatore a stravolgere le ipotesi formulate all'inizio (fig. 3).

Figura 3

\section{Sviluppo della competenza comunicativa}

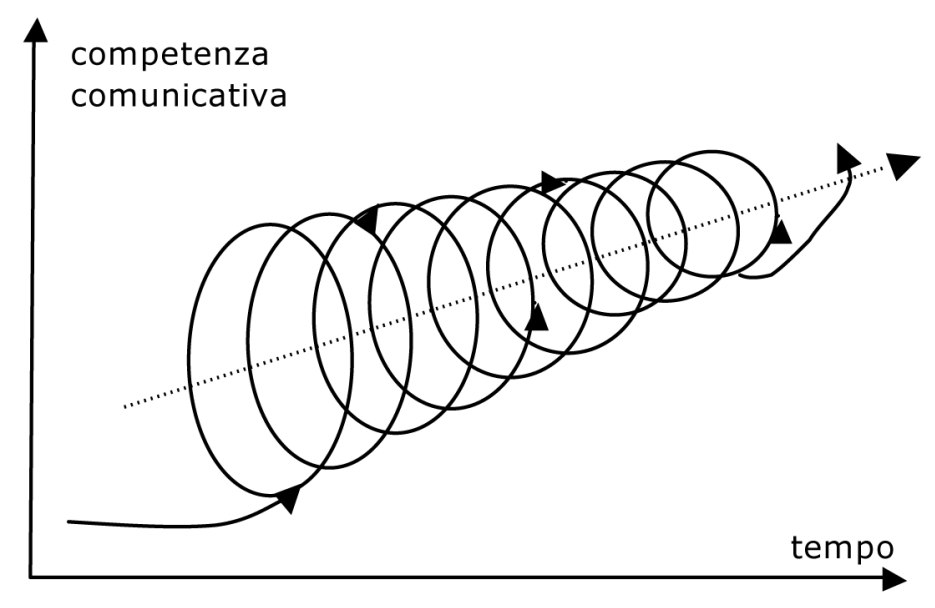

Il processo di costruzione della competenza comunicativa è elicoidale; la linea che ingenuamente si sarebbe tentati di scorgere non è altro che l'asse (astratto) di un vortice che procede per ritorni su quanto appreso, in una sequenza ininterrotta di validazioni e proceduralizzazioni. 


\section{Punto c}

Si scrive e si parla, sostengono Vigil \& Oller, spinti da (1976: 289)

un profondo bisogno di definire il proprio io, e di trovare accettazione nell'esprimere questo io in rapporto con altri di cui si ha stima. La lingua, quindi, serve anche come base per la definizione degli io altrui e per il rafforzamento reciproco di identità sia individuali che collettive.

I due studiosi mettono in evidenza le variabili affettive coinvolte nell'atto del comunicare, sottolineando il valore pragmatico ed espressivo della lingua. Sulla base della percezione di significatività o meno dell'interazione, un soggetto addirittura (1976: 289-290; la traduzione dall'inglese è di Humphris, 2005)

può decidere di inviare un messaggio oppure no, e se lo fa può decidere che cos'è che vuole inviare [...]. La fonte non solo crea il messaggio e lo invia ma crea un costrutto ipotetico circa la probabile reazione dell'interlocutore nei confronti del messaggio.

In altre parole, gli atti comunicativi che si producono sono sensibili alla relazione che il mittente ha con il destinatario: questa determina il colore affettivo che l'output avrà; in alcuni casi, il primo può anzi decidere che non si dia alcuna produzione o che essa sia ridotta al minimo.

Laddove, invece, il rapporto tra i due sia significativo, comunicare permette di creare e ricreare se stessi (nel dirla con Bergson); di trovare nell'altro uno specchio per cogliere bisogni che altrimenti non sarebbero visibili.

In sintonia con Vigil e Oller e con l'intera tradizione umanista (cfr. Stevick 1990; Dufeu 1996; Arnold 1999; Rinvolucri 1999), la PAC recupera la dimensione affettiva del comunicare, pressoché ignorata sia dagli strutturalisti che dai comunicativisti. Per lo strutturalismo, infatti, l'affettivo ricopriva un ruolo ancillare: l'insegnante si prodigava per addolcire la pillola degli esercizi ripetitivi, creando una cornice ludica (con giochi, drammatizzazioni, ecc., cfr. Humphris 2006). Nel comunicativismo è vero, certo, che si parla di "filtro affettivo" (cfr. Krashen 1985) ma che cosa sia e da cosa sia scatenato sono questioni su cui si stende la nebbia più fitta.

Ricapitolando quanto detto: le abilità produttive vanno riabilitate dal momento che è per loro tramite che si stabilizza la grammatica interna; inoltre, se esse si esercitano sui contenuti di una lettura o di un ascolto, la comprensione ne esce approfondita e strutturata (bellissimi esempi di integrazione delle abilità in Stephan 2006). Non va dimenticato che l'espressione scritta/orale è occasione di socializzazione e di autopromozione: chi parla si rivela, ha modo di esprimere desideri e aspirazioni; riconosce e condivide le esperienze altrui. La lingua, così, acquista in significatività. 


\section{Promuovere le abilità produttive}

Una volta considerata l'importanza delle abilità produttive, rimane da tracciare una pista metodologica.

Promuovere l'interazione orale e la scrittura significa, in primo luogo, sostenere l'apprendente di fronte alle difficoltà dovute ad un dominio parziale del codice.

Scrive Humphris (2008):

A causa del suo linguaggio ancora molto rozzo rispetto alla complessità del suo pensiero, lo studente si espone continuamente a fare figuracce. La probabilità che venga visto diverso da ciò che ritiene di essere è molto alta. Tramite la sua prima lingua ha messo parecchi anni a mettere a punto una propria identità. Nella propria lingua, nel proprio ambiente, viene considerato magari spiritoso, o generoso, o intelligente, o simpatico, o solare, o saggio, o... Con la lingua nuova non c'è nessuna garanzia che posso risultare tale agli occhi degli altri. Dal punto di vista dello studente è una strategia intelligente, quindi, cercare di ridurre tale rischio. È intelligente, quindi, cercare di accorciare i tempi in cui il rischio è più alto, cioè i momenti in cui la richiesta dell'insegnante è «nuotare in alto mare senza salvagente», cioè le produzioni libere.

Per motivare gli studenti alla produzione si possono creare condizioni di pressione comunicativa, di vuoto di informazione oppure si possono personalizzare gli scambi.

Analizziamo le tre azioni didattiche nei paragrafi che seguono (per approfondimenti, cfr. Torresan 2014a).

\subsection{La pressione comunicativa}

Per pressione comunicativa intendiamo una serie di pratiche che forzano lo studente a produrre lingua: lo mettono nelle condizioni di dire qualcosa o di scrivere su qualcosa.

Alcune attività volte a promuovere la fluenza che giocano sul fattore tempo costituiscono ottimi esempi di pressione comunicativa.

Due esempi a riguardo sono la scrittura veloce e l'interazione in cerchi concentrici.

Nella prima -presentata da Rinvolucri (2001: 95; Arnold et al. 2005: 9192), su adattamento di un'idea di Klauser (1987) - gli studenti, dopo una breve attività di movimento, sono chiamati alla stesura automatica dei loro pensieri, su un tema deciso dall'insegnante (es.: "ieri"; "cosa avrei fatto oggi se non fossi stato/a qui”, ecc.), all'interno di un tempo stabilito. L'attività può risultare proficua per coloro che si sentono preda del blocco dello scrittore, del panico di fronte al foglio intonso: "90 secondi di scrittura automatica sono migliori di dieci minuti in cui lo studente morde la penna", aspettando l'ispirazione che non giunge (Rinvolucri 2002: 95). 
In Hess 2001 (54-56) si descrive invece l'attività dei cerchi concentrici. Gli studenti sono divisi in due cerchi, concentrici, in maniera tale che a uno studente del cerchio interno ne corrisponda uno del cerchio esterno. Gli studenti dialogano con il partner su un tema deciso dall'insegnante per due minuti. Successivamente un cerchio viene fatto girare e si formano nuove coppie.

Anche attività di improvvisazione generano pressione comunicativa: chi improvvisa è chiamato a reagire in tempo rapido allo stimolo offerto dal compagno (cfr. Wilson 2008; Serafini, Zanardi 2014).

Inoltre, si ha pressione comunicativa ogni qualvolta i partecipanti di un evento comunicativo perseguono scopi contrastanti, ovvero sono posti in una condizione di conflitto comunicativo (cfr. Torresan 2014b).

In Beltramo (2000), per esempio, si descrive un'attività di scrittura in cui ogni studente deve difendere i propri interessi a scapito di quelli degli altri. Dopo aver scelto un animale con cui identificarsi, lo studente immagina di trovarsi di fronte all'arca di Noè; il diluvio sta per iniziare però nell'arca c'è posto per un solo animale. Al fine di essere ammesso sull'arca, l'allievo deve scrivere una lettera all'insegnante (il quale veste i panni di Noè) in cui illustra le qualità dell'animale da lui rappresentato e scredita gli altri animali.

In Catizone et al. (1999) la competizione promuove un confronto serrato tra coppie. È il cosiddetto gioco dell'alibi, che descriviamo nel riquadro sottostante (il testo è adattato).

\section{IL GIOCO DELL'ALIBI}

Gli studenti vengono divisi a coppie: da un lato i sospettati, dall'altro i poliziotti (nel rapporto di $2: 1$ ).

L'insegnante scrive alla lavagna "Ieri alle .... qualcuno ha ....", e completa l'annuncio con l'indicazione dell'ora e del reato commesso.

Ai sospettati aggiunge: "Dovete trovare un alibi. La polizia vi interrogherà separatamente e le vostre descrizioni dovranno combaciare".

Ai poliziotti (che vengono informati dall'insegnante sui luoghi concordati dalle coppie, es: ristorante, cinema, teatro, ecc.) annuncia: "Dovete formulare domande dettagliate e l'interrogato non può rispondere di non sapere o di non ricordare".

Dopo che le coppie si sono accordate sull'alibi e i poliziotti si sono confrontati circa le domande da realizzare, ha inizio l'interrogatorio.

Ogni poliziotto interroga contemporaneamente membri di due o tre coppie diverse; quindi, successivamente, si confronta con il collega che ha interrogato i membri delle stesse coppie.

I poliziotti stabiliscono infine, sulla base delle discrepanze riscontrate tra le versioni, qual è la coppia colpevole.

Per Humphris, una iniziativa che, nella logica di molti insegnanti, vorrebbe facilitare la produzione -la presentazione di un ampio ventaglio di temi- rischia di sortire un effetto negativo, trasformando l'attività di dialogo in un mero esercizio (2008): 
Spesso l'insegnante, volendo aiutare gli studenti ad avere tante cose da dire, gonfia il tema. Esempio: le vacanze. "Parlate delle ultime vacanze, delle prossime vacanze, delle vacanze che sognate, della più bella vacanza che avete fatto; parlate dei luoghi, delle persone che avete conosciuto, dei mezzi di trasporto, del tempo, dell'alloggio, di tutto quello che volete». In realtà, questo «banchetto» tematico produce l'effetto opposto a quello che l'insegnante voleva. Non ha un effetto «ispiratore», non invoglia lo studente a cominciare a parlare di qualcosa, lo induce, invece, a perdere tempo a pensare «quale mi piace di più?» o meglio «quale mi annoia di meno?». Oppure ci sono quegli studenti che pedissequamente percorrono in ordine l'elenco dei temi, parlando un po' di ognuno, come se fosse un esercizio controllato.

A detta del metodologo inglese (romano di adozione), il problema nasce dal fatto che l'insegnante non riconosce la capacità dello studente di farcela da solo:

Dov'è l'errore dell'insegnante? Di nuovo, secondo me, siamo di fronte ad una carenza di fiducia verso gli studenti. Se l'insegnante ha realmente fiducia sa che tutti gli studenti (perlomeno tutti quelli che studiano la lingua da almeno 100 ore), sono in grado di parlare con un altro studente per più di mezz'ora delle ultime vacanze. E sono in grado di fare altrettanto riguardo alle prossime vacanze. E idem per le vacanze che sognano. E idem per la più bella vacanza che hanno fatto. Insomma 4 temi che dovevano essere argomenti di 4 produzioni libere orali diverse vengono proposti tutti insieme. Non c'è niente da fare: non riusciamo a nascondere le nostre ansie allo studente. La fiducia nelle capacità dello studente da parte dell'insegnante che propone un solo tema viene percepita dallo studente. Come viene percepita la mancanza di tale fiducia da parte dell'insegnante che offre un «banchetto» di temi. E lo studente risponde a tono. Nel secondo caso, la consegna viene vista come un noioso compito scolastico. Nel primo caso, invece, parla di una sola cosa, e man mano procede nel racconto, i fatti, le emozioni tornano sempre più presenti in mente e la curiosità dell'interlocutore viene a poco a poco maggiormente sollecitata. Il risultato è una conversazione sempre più interessante per i due partecipanti, i quali, di conseguenza, si impegnano sempre di più a cercare di capirsi.

Oltre, dunque, al fattore tempo e al fattore competizione, pure l'essenzialità delle consegne concorre a determinare pressione comunicativa.

\subsection{Il vuoto di informazione}

C'è vuoto di informazione quando il parlante condivide con l'interlocutore quello che fino a poco tempo prima era una sua informazione privata.

Molti testi di metodologia promuovono situazioni di gap comunicativo ben definite: compiti a carattere predittivo (a uno studente mancano dei dati per 
completare il profilo del compagno) e, con assai più frequenza, attività di discriminazione tra realtà e immaginazione.

Un esempio a riguardo del primo caso è una produzione scritta presentata in Frank, Rinvolucri (2007): uno studente è tenuto a indovinare una caratteristica del compagno o una situazione che questi ha vissuto, sulla base di alcuni indizi. La scansione delle fasi, qui leggermente adattate, è la seguente:

- Pensa ad un fatto accaduto in particolare. Pensalo nei dettagli.

- Scrivi 10 domande di comprensione in ordine cronologico; passale al compagno;

- Il compagno è tenuto a sviluppare un testo che contenga le risposte (immaginarie) a quelle domande;

- Una volta scritto il testo, il compagno si confronta con te sulla veridicità delle sue ipotesi e tu fai altrettanto.

Un’attività simile è quella pensata da Beatriz Dállia (2009):

- Si divide la classe in gruppi; a ciascuno studente si consegna un foglio bianco.

- A turno ciascuno racconta qualcosa di sé (per esempio un ricordo di scuola). Gli altri, nel frattempo prendono nota, cercando di intuire, sulla base del racconto, le caratteristiche psicologiche di chi racconta.

- L'attività si conclude con un confronto: si possono correggere, precisare o confermare le ipotesi dei compagni.

Per quanto riguarda, invece, il caso in cui gli studenti si debbano confrontare con dati falsificati, citiamo un'attività tratta da Delle Donne (1996) 5 .

\section{DUE BUGIARDI}

Tre volontari vengono fatti uscire dall'aula: si devono accordare per raccontare un fatto.

Uno di loro racconterà un fatto realmente accaduto, gli altri racconteranno lo stesso fatto (come se l'avessero vissuto pure loro) però con delle piccole differenze.

Gli altri gruppi ascolteranno i tre racconti e dovranno decidere chi dice la verità: hanno la possibilità di fare tre domande a ciascuno dei tre membri.

\subsection{Personalizzazione}

Posto che l'interlocutore sia disposto ad ascoltare e che tra i poli della comunicazione, emittente e destinatario, si sia instaurato un clima di fiducia e di rispetto o che, più in generale, all'interno della classe abbiano avuto modo di generarsi processi di appartenenza (group-bonding), l'interazione può vertere su contenuti personali. Lo studente rivela qualcosa di sé all'altro e la comunicazione ne acquista in significatività. 
Rinvolucri precisa le condizioni per introdurre attività personalizzate -da lui chiamate humanistic exercises (1999: 197-8, la traduzione è nostra):

Un esercizio umanistico può essere usato in classe qualora l'insegnante abbia una consapevolezza profonda delle dinamiche di gruppo e di come queste influenzino l'apprendimento. Egli [...], nel momento in cui pianifica le lezioni, tiene conto dell'atmosfera presente in classe [...]; è consapevole di insegnare a quaranta individui, non già a una massa indistinta; da buon osservatore, è capace di ascolto empatico.

L'insegnante personalizza ogni volta che promuove l'esercizio della funzione personale della lingua. Quest'ultima, scrive Balboni (2008²: 76; il corsivo è nostro),

si realizza quando lo studente rivela la propria soggettività, la propria personalità, quando manifesta sentimenti, emozioni, pensieri, impressioni, sensazioni [...]. Molti materiali didattici, improntati a una mera comunicazione pragmatica, attribuiscono un peso piuttosto limitato a questa funzione, fondamentale sul piano dell'affettività e della motivazione.

Altrove illustrammo la complessità che la personalizzazione può comportare, in termini metodologici, visti i molteplici oggetti su cui può vertere la comunicazione: io, l'altro, gli altri (Torresan 2011).

Si può parlare di sé direttamente oppure mediante proiezione (tra le altre fonti, cfr. Rinvolucri 2002; Heathfield 2005; Taylor 2001; Montali, Mazza 2000; Sion 2001).

Si può passare da temi piuttosto generali, come:

un pericolo
un nonno
il mio amico
un sogno
un mio insegnante
il libro

a questioni più specifiche:

il mio rapporto con l'acqua tre passeggiate memorabili che ho fatto il ritrovamento di un oggetto smarrito come ho cominciato a coltivare un hobby/interesse

un regalo ricevuto cosa facevo ieri a quest'ora quindici minuti della giornata di ieri una telefonata ricevuta ieri

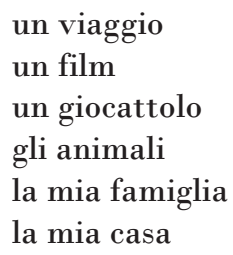

la storia dei miei capelli un albero la via per raggiungere la scuola un no che ho detto recentemente io e la musica il mio modo di rilassarmi una storia di successo io e l'apprendimento dell'italiano una decisione importante presa in tempi recenti 
Non tutti i metodologi condividono l'appello all'essenzialità delle consegne avanzato da Humphris al fine di generare pressione. Laddove, anziché descrivere un fatto che si presti ad essere raccontato in maniera vivida (come le vacanze), lo studente è chiamato all'introspezione, non è raro imbattersi in liste che valgono a sostegno della conversazione.

La seguente, per esempio, incentrata sul mal di testa, è tratta da Davis et al. (1998: 19):

- Ti capita di avere mal di testa?

- Spesso?

- In quale momento della giornata?

- Quando dura?

- Quand'è che hai avuto l'ultimo?

- In quale parte della testa avverti il mal di testa?

- Che tipo di dolore è?

- Come fai a capire che il mal di testa non c'è più?

- Trovi che la gente ti possa compatire quando hai mal di testa?

- Quale di queste parole ti paiono adatte per descrivere un mal di testa? violento; assordante; martellante; lento; verde; pesante; oscuro; acuto; persistente;

- Altre persone della tua famiglia soffrono di mal di testa?

Degli stessi autori è la scheda che segue, somministrata per mezzo di un dettato; ha per oggetto l'atto del fantasticare (14-15):

- Sogno spesso ad occhi aperti?

- Quante volte al giorno?

- Più al mattino o verso sera?

- Dove mi capita più spesso di sognare ad occhi aperti?

- Quanto durano i miei sogni ad occhi aperti?

- Quando comincio a sognare ad occhi aperti?

Lo studente è quindi invitato a stendere altre due domande di suo pugno sullo stesso tema. Quindi riprende a scrivere sotto dettatura:

- In quale modo ritorno al mio stato normale di concentrazione?

- Sognando mi aspetto di ottenere qualche risultato?

- Perché sogno ad occhi aperti?

- C'è qualche differenza, secondo me, tra il sognare ad occhi aperti e il pensare? Quali sono le differenze?

- C'è un aspetto di pericolo nei miei sogni ad occhi aperti?

- Ho sognato ad occhi aperti durante questo dettato? 
Si badi: qualora l'insegnante si senta maggiormente a suo agio con ricognizioni più immediate e 'leggere', può ricorrere a schede meno impegnative, prodotte, al limite, da lui stesso. Un esempio, a tal proposito, è il dettato-questionario (cfr. Taylor 2001): l'insegnante detta delle frasi e gli studenti le trascrivono solo se sono vere per loro. A trascrizione avvenuta, si confrontano a coppie o in piccoli gruppi per controllare l'ortografia e scambiarsi informazioni su gusti e abitudini. Si legga l'esempio che segue:

- L'italiano è difficile

- Arrivo spesso in ritardo

- Non mi piacciono le persone che parlano molto

- Scrivo molte email

- Non sopporto le giornate di pioggia

- Leggo prima di dormire

- Mi arrabbio spesso

- Sono una persona ordinata

- Odio i gatti

- Parlo più di due lingue

- Qualcuno dice che sono lento/a

- Mi piace alzarmi molto presto

Una scheda che vale da guida, a nostro parere, può tornare utile anche qualora le produzioni personalizzate facciano leva su processi proiettivi, quando lo studente, cioè, parla di sé mediante un oggetto interposto (cfr. Manes 1997, 2007). Una lista di controllo, in tal caso, viene in soccorso soprattutto a coloro che si reputano poco o affatto creativi. Proponiamo come esempio questa composizione guidata, consegnata come compito per casa a studenti di livello B1, dopo che avevano lavorato in classe su una canzone dei Lunapop: Vespa Special 50 (siamo grati a Beatrice Giudice per averci suggerito l'idea):

Immagina di essere il protagonista della canzone e scrivi una lettera alla tua Vespa. Devi:

- ringraziarla per tutto il tempo che è stata insieme a te;

- ricordarle i bei viaggi fatti;

- ricordarle l'incidente in cui vi siete trovati coinvolti, senza però subire danni né riportare lesioni;

- chiedere perdono se qualche volta ti sei dimenticato di pulirla;

- dirle che dovrai cambiarla, perché ti compri una macchina.

- salutarla.

Non è detto che lo scrivente abbia familiarità con l'oggetto prescelto (nel nostro caso, non tutti gli studenti posseggono un ciclomotore), tuttavia è importante che l'esempio evochi un'esperienza universale (nel caso sopra, il congedo da qualcosa di caro). 
Da ultimo, ci piace menzionare una tecnica di Rinvolucri 'One Question Many Answers' (Frank, Rinvolucri 1991). Essa consiste in una serie di domande che uno studente rivolge ripetutamente all'altro: mano a mano che l'interrogazione procede, la conversazione si sposta dal dato superficiale ad aspetti meno visibili, ad analogie personali, a ricordi ed associazioni ${ }^{6}$.

- Cos'è un vulcano?

Una montagna con un buco in mezzo.

- Cos'è un vulcano?

L'Etna è un vulcano.

- Cos'è un vulcano?

Una potenza che può distruggere le cose intorno.

- Cos'è un vulcano?

Un fenomeno collegato ai terremoti.

- Cos'è un vulcano?

Una montagna da cui esce lava.

- Cos'è un vulcano?

Io sono un vulcano quando mi arrabbio.

\section{Conclusioni}

In questo articolo abbiamo sostenuto che le abilità produttive concorrono al potenziamento della competenza metalinguistica e abbiamo dichiarato che esse, se associate ad attività di comprensione, permettono un approfondimento dei significati, a vantaggio di una maggiore esplorazione del testo e di una più cospicua ritenzione delle informazioni.

Inoltre, abbiamo precisato, parlare e scrivere fanno assumere all'allievo un ruolo proattivo e creativo nei confronti della lingua.

Affinché lo studente si trovi nelle condizioni di superare il naturale timore verso un codice che possiede solo in parte e che, dunque, gli appare come uno strumento limitato e limitante (dal momento che non gli permette di esprimersi come vorrebbe), l'insegnante può allestire un contesto comunicativo che renda lo scambio soggetto a pressione, che soddisfi il bisogno naturale di sapere e che faccia ricorso alla funzione personale della lingua.

\section{Note}

1 "Capacità di descrivere i meccanismi di funzionamento della lingua"; ovverosia di definire la natura grammaticale di una parola o di un'espressione e di rappresentare la relazione tra forme linguistiche (per esempio: imperfetto e passato prossimo), cf. Balboni 1999 (45).

2 Si legge in Balboni (1999: 40): "Il termine è proprio della lessicografia, dove ad esempio descrivono le metafore fossilizzate, usate cioè senza che ci si renda conto che si 
tratta di metafore («fossilizzazione» e «rendere conto», ad esempio, sono due metafore fossilizzate). II termine è stato ripreso in glottodidattica per indicare errori che vengono ripetuti costantemente, per cui l'errore si fissa e diviene particolarmente difficile da superare".

3 Alcune ricerche dimostrano, infatti, come l'assenza di una retroalimentazione possa ostacolare il processo di acquisizione. Studenti francofoni e italofoni di inglese continuano a produrre frasi con costruzioni avverbiali posposte al verbo (He goes quickly into che kitchen*), calcate sulla sintassi della LM, nonostante siano inondati di esempi in cui l'avverbio è preposto (He quickly goes into che kitchen). II fatto che nell'input non siano presenti esempi della struttura che producono non li porta a considerare l'erroneità della loro ipotesi (Trahey, White 1993).

Ulteriori ricerche confermano questi dati. Williams e Evans (1998) hanno messo a confronto la condotta linguistica di due gruppi. I gruppi venivano 'inondati' di input ricchi di forme da acquisire (input flood), tuttavia solo in uno dei due l'insegnante era autorizzato a correggere: alla fine, i risultati che quest'ultimo gruppo otteneva in termini di accuratezza erano nettamente superiori a quelli dell'altro.

4 Per approfondimenti sulle caratteristiche dei task comunicativi che maggiormente incidono sulla complessità dell'output, cfr. Long 1989; Coonan 2002.

5 Per approfondimenti sulle strategie che generano vuoto di informazione rimandiamo alla raccolta di attività presenti in Heathfield 2005.

6 L'attività insiste maggiormente sulla consapevolezza intrapersonale nel caso in cui le domande (o le affermazioni che valgono da stimolo) abbiano a che fare con gli affetti:

Chi sei?

I tuoi amici

Dove vivi?

Cosa ti piace?

Cosa non ti piace?

Una cosa che ti piace dell'Italia (per chi ha avuto modo di visitare l'Italia).

\section{Bibliografia}

Arnold J., 1999 (a cura di), Affect in Language Learning, Cambridge University Press, Cambridge.

Balboni P. E., 1994, Didattica dell’italiano a stranieri, Bonacci, Roma.

Balboni P. E. 1997, "Tecniche di didattica grammaticale", in Cambiaghi B. (a cura di), La didattica della grammatica, CLUC-La Scuola Editrice, 101-114.

Balboni P. E., 1999, Dizionario di glottodidattica, Guerra-Soleil, Perugia-Welland.

Balboni P. E., 2007, Modelos operativos para la educación lingüística, Guerra, Perugia.

Balboni P. E., 2008², Le sfide di Babele, Utet, Torino.

Beltramo A., 2000, Abilità di scrittura, Paravia, Torino.

Brumfit C., Johnson K., 1978 (a cura di), The Communicative Approach to Language Teaching, Oxford University Press, Oxford. 
Caon F., 2005, Un approccio umanistico affettivo all'insegnamento dell'italiano a non nativi, Cafoscarina, Venezia.

Catizone P., Humphris C., Micarelli L., 1999, Volare 2, Dilit, Roma.

Coonan M. C., 2002, La lingua straniera veicolare, Utet, Torino.

Dabrowska E., 2004, Language, Mind and Brain. Some Psychological and Neurological Constraints on Theories of Grammar, Georgetown University Press, Washington D.C.

Dal Forno I., 1999, Tocca a te, Guerra, Perugia.

Dállia B., 2009, "Sai chi sono io?", Bollettino Itals, 31, < www.itals.it >.

Davis P, Garside B., Rinvolucri M., 1998, Ways of Doing, Cambridge University Press, Cambridge.

Delle Donne E., 1996, L'italiano praticamente, Guerra, Perugia.

Dufeu B., 1994, Teaching Myself, Oxford University Press, Oxford.

Ellis R., 1998, "Teaching and Research. Options in Grammar Teaching”, TESOL Quarterly, 32, 39-59.

Ferencich R., Torresan P., 2005, Giochi senza frontiere, Attività ludiche per l'insegnamento dellitaliano, Alma, Firenze.

Frank C., Rinvolucri M., 1991, Grammar in Action Again, Prentice Hall International.

Frank C., Rinvolucri M., 2007, Creative Writing: Activities to Help Students Produce Meaningful Texts, Helbling, Innsbruck.

Freddi G., 1979, Didattica delle lingue moderne, Minerva Italica, Bergamo.

Guastalla C., 2008, "Il Due chiacchiere con Christopher Humphris", <ildueblog. it>.

Heathfield D., 2005, Spontaneous Speaking, Delta, Peaslake.

Humphris C., 1982a, "La lettura", Bollettino Dilit, $2<$ www.dilit.it >

Humphris C., 1982b, "La colmatura in funzione", Bollettino Dilit, $2<$ www.dilit. it $>$

Humphris C., 1982c, "Una lezione di lettura: un esempio", Bollettino Dilit, $2<$ www.dilit.it >

Humphris C., 1988, "La lettura e la sicurezza di sé", Bollettino Dilit, $3<$ www. dilit.it >

Humphris C., 2005, "Introduzione al XV seminario internazionale per insegnanti di lingua 2005", <www.dilit.it>.

Humphris C., 2008, "Produzione libera", Bollettino Dilit, 1, <www.dilit.it>.

Izumi S., 1999, "Testing the Output Hypothesis. Effects of Output on Noticing and Second Language Acquisition", Studies in Second Language Acquisition, $21,3,421-452$.

Izumi S., 2002, "Output, Input Enhancement, and the Noticing Hypothesis. An Experimental Study on ESL Relativiation", Studies in Second Language Acquisition, 24, 4, 541-577.

Klauser H. A., 1987, Writing on Both Sides of the Brain: Breakthrough Techniques for People Who Write, Harper Collins, New York.

Krashen S. D., 1981, Second Language Acquisition and Second Language Learning, Pergamon Press, Oxford. 
Krashen S. D., 1983, Principles and Practice in Second Language Acquisition, Pergamon Press, Oxford.

Krashen S. D., 1985, The Input Hypothesis, Longman, London.

Long M. H., 1989, “Tasks, Groups and Task-Group Interactions”, University of Hawai Working Papers in ESL, 8, 2.

Long M. H., 1991, "Focus on Form: A Design Feature in Language Teaching Methodology", in de Bot K.; Ginsberg R. B.; Kramsch C. (a cura di), Foreign Language Research in Cross-cultural Perspective, John Benjamins Publishing Company, Amsterdam, 39-52.

Long M. H., 1996, "The Role of the Linguistic Environment in Second Language Acquisition", in Ritchie, W. \& Bhatia, T. K. (a cura di), Handbook of Second Language Acquisition, New York Academic Press, 413-468.

Manes S., (a cura di), 1997, 83 giochi psicologici per la conduzione dei gruppi, Franco Angeli, Milano.

Manes S., (a cura di), 2007, Giochi per crescere insieme, Franco Angeli, Milano.

McLaughlin B., 1987, Theories of Second Language Learning, Arnold, Londra.

Montali S., Mazza A., 2000, Parlando parlando, Alpha Beta, Bolzano.

Nunan D., 2001, Second Language Teaching and Learning, Heinle \& Heinle, Boston.

Pallotti G., 2005, "Le ricadute didattiche delle ricerche sull'interlingua", in Jafrancesco E. (a cura di), L'acquisizione dell'italiano L2 da parte di immigrati adulti, Edilingua, Atene, 43-59.

Pallotti G., 2002, "A cosa serve insegnare una lingua? Qualche riflessione a partire da Volare", in Humphris C. (a cura di), Per una pratica didattica coerente. Atti del XIII seminario internazionale per insegnanti di lingua, Roma, 4-6 maggio 2001, Dilit, Roma, 13-27.

Puchta H., Rinvolucri M., Arnold J., 2007, Imagine That!, Cambridge University Press, Cambridge.

Rinvolucri M., 1999, "The Humanistic Exercise", in Arnold J. (a cura di), Affect, 194-210.

Rinvolucri M., 2002, Humanising Your Coursebook, Delta, Peaslake.

Schmidt R., 1990, "The Role of Consciousness in Second Language Learning", Applied Linguistics, 129-158.

Serafini, C.; Zanardi, M., 2014, "Role-play e improvvisazione teatrale. Tecniche al servizio della competenza comunicativa e interculturale nella didattica dell'italiano LS", Bollettino Itals, 12, 55 <www.itals.it>.

Sion C., 2001, Creating Conversation in Class, Delta, Peaslake.

Stephan, S., 2006, "Personalizing Language", Humanizing Teaching Language Journal, 11, 5 <www.hltmag.co.uk>.Stevick E. W., 1990, Humanism in Language Teaching, Oxford University Press, Oxford.

Swain M., 1985, "Communicative Competence: Some Roles of Comprehensible Input and Comprehensible Output in its Development", in Gass, S. \& Madden C. (a cura di), Input in Second Language Acquisition, Newbury House, Rowley, 235-253. 
Swain M., 1991, "Manipulating and Complementing Content Teaching to Maximize Second Language Learning", in Phillipson R. et al. (a cura di), Foreign / Second Language Pedagogy Research, Multilingual Matters, Clavedon.

Taylor J., 2001, The Minimax Teacher, Delta, Peaslake.

Torresan, P., 2011, “«Come barchette di carta su un mare di emozioni»: personalizzare l'insegnamento dell'italiano", Cuadernos de italianistica cubana, 7, 28-42.

Torresan, P. (ed.), 2014a, Didáctica de la expresión oral en la clase de idiomas, Istituto Italiano di Cultura, Lima.

Torresan, P., 2014b, "Consensus gap, info gap, conflitto e pressione comunicativa", Officina.it, 46, <www.almaedizioni.it>.

Trahey M., White L., 1993, "Positive Evidence and Pre-emption in the Second Language Classroom", Studies in Second Language Classroom, 181-204.

Vigil N. A., Oller J. W., 1976, "Rule Fossilization: A Tentative Model”, Language Learning, 26, 281-295.

Wilson K., 2008, Drama and Improvisation, Oxford University Press, Oxford. 